\title{
Effect of Isometric Hip Abduction on Foot and Ankle Muscle Activity and Medial Longitudinal Arch During Short-Foot Exercise in Individuals With Pes Planus
}

\author{
Jung-Hoon Choi, Heon-Seock Cynn, Chung-Hwi Yi, Tae-Lim Yoon, and Seung-Min Baik
}

\begin{abstract}
Context: The improvement of hip joint stability can significantly impact knee and rearfoot mechanics. Individuals with pes planus have a weak abductor hallucis ( $\mathrm{AbdH})$, and the tibialis anterior (TA) may activate to compensate for this. As yet, no studies have applied isometric hip abduction (IHA) for hip stability during short-foot exercise (SFE). Objective: To compare the effects of IHA on the muscle activity of the AbdH, TA, peroneus longus (PL), and gluteus medius (Gmed), as well as the medial longitudinal arch (MLA) angle during sitting and standing SFE. Design: Two-way repeated analyses of variance were used to determine the statistical significance of AbdH, TA, PL, and Gmed electromyography activity, as well as the change in MLA angle. Setting: University research laboratory. Participants: Thirty-two participants with pes planus. Intervention(s): The participants performed SFE with and without isometric hip abduction in sitting and standing positions. Main Outcome Measures: Surface electromyography was used to measure the activity of the AbdH, TA, PL, and Gmed muscles, and Image J was used to measure the MLA angle. Results: Significant interactions between exercise type and position were observed in terms of the PL muscle activity and in the change in MLA angle only, while other muscles showed significant main effects. The IHA during SFE significantly increased the AbdH muscle activity, while the TA muscle activity was significantly lower. The muscle activity of Gmed and PL was significantly increased in the standing position compared with sitting, but there was no significant difference with or without IHA. The change in the MLA angle was significantly greater in SFE with IHA in a standing position than in the other SFE conditions. Conclusions: IHA may be an effective method for reducing compensatory TA activity and increasing AbdH muscle activity during SFE for individuals with pes planus.
\end{abstract}

Keywords: flat foot exercise, foot intrinsic muscle strengthening, foot stability exercise, hip stability, medial longitudinal arch of foot

Pes planus, a pronated foot, is often attended by a calcaneal eversion and abducted forefoot, as well as flattening or lowering of the medial longitudinal arch (MLA). ${ }^{1}$ During weight-bearing activities, the foot is stabilized by maintaining the MLA via passive support (bony structure and ligamentous) and active support (extrinsic and intrinsic foot muscles), and it is used as a rigid lever that allows muscular contraction to propel the body..$^{1,2}$ In particular, intrinsic foot muscles, such as the abductor hallucis (AbdH), flexor hallucis brevis, flexor digitorum brevis, and interosseous muscles, provide critical stabilization of the foot arch during propulsion. ${ }^{3}$ Excessive pronation, represented by a lowering of the MLA, is associated with a number of pathological conditions involving the lower extremities, including plantar fasciitis, ${ }^{4}$ hallux valgus, ${ }^{5}$ Achilles tendonitis, ${ }^{6}$ and patellofemoral pain syndrome. ${ }^{7}$

Several exercises, such as picking up objects, toe towel curls, shin curls, and sitting short-foot exercise (SFE), have been used to strengthen foot muscles and prevent lowering of the MLA, as well as improving foot stability. ${ }^{8-10}$ SFE, an effective method of

Choi, Cynn, and Baik are with the Applied Kinesiology and Ergonomic Technology Laboratory, Department of Physical Therapy, The Graduate School, Yonsei University, Wonju, Gangwon-do, Republic of Korea. Choi is also with the Department of Rehabilitation Team, Yongin Severance Hospital, Yongin-si, Gyeonggi-do, Republic of Korea. Yi is with the Department of Physical Therapy, The Graduate School, Yonsei University, Wonju, Gangwon-do, Republic of Korea. Yoon is with the Department of Physical Therapy, College of Health \& Medical Sciences, Cheongju University, Chungcheongbuk-do, Korea. Cynn (cynn@yonsei.ac.kr) is corresponding author. recruiting the $\mathrm{AbdH}$ and preventing excessive lowering of the MLA, is often prescribed and performed in the sport and rehabilitation field. ${ }^{8}$ In addition, SFE has been studied in participants with pes planus, in various conditions. Previous studies have compared the muscle activity of the $\mathrm{AbdH}$ and tibialis anterior (TA), as well as the MLA angle, between neutral foot participants and pes planus participants during SFE in a sitting position..$^{10}$ According to the results, AbdH activity was significantly lower and TA/AbdH ratio activity was significantly greater in the pes planus group than in the neutral foot group. ${ }^{10}$ In a study comparing the immediate effect of SFE in sitting and standing positions on dynamic balance, SFE was found to significantly increase the limit of stability. ${ }^{11}$ Furthermore, Jung et $\mathrm{al}^{2}$ compared the AbdH activity and MLA angle between the toe curl exercise and SFE in both sitting and one-leg standing positions. The results indicated that AbdH activity in SFE was significantly greater than that in the toe curl exercise for both exercise postures. In addition, the MLA angle in the SFE was significantly smaller than in the toe curl movement for both postural positions. ${ }^{2}$ According to a study comparing the muscle activity of $\mathrm{AbdH}, \mathrm{TA}$, and peroneus longus (PL) during SFE at various ankle angles, SFE performed when the ankle was neutral and during dorsiflexion showed a higher TA and $\mathrm{AbdH}$ muscle activity than SFE at ankle plantarflexion. ${ }^{12}$

Weakness or dysfunction of the hip external rotator can lead to hip adduction and medial rotation and dynamic knee valgus, which can eventually affect the foot pronation. ${ }^{1,13}$ During running, the significant increase in hip strength results in a significant reduction in the foot eversion range of motion, a trend toward reduced hip 
medial rotation range of motion, and significant decreases in knee abduction and ankle inversion moment. ${ }^{14}$ Several studies have reported that the weakness or dysfunction of the hip is not only a cause of adjacent knee injuries, such as patellofemoral pain syndrome and noncontact anterior cruciate ligament injury, but it also causes ankle and foot injuries, such as chronic ankle sprain and plantar fasciitis. $4,13,15$ Therefore, the improvement of hip joint stability through the strengthening of hip muscles can have a significant impact on knee and rearfoot mechanics, which can ultimately improve the dynamic alignment of the lower limbs. ${ }^{14}$

Although previous studies have shown that hip muscular strengthening and foot intrinsic strength exercises were effective for ankle and foot stability, no previous studies have applied isometric hip abduction (IHA) for hip stability during SFE. During SFE, the stability of the hip provided by applying IHA will affect the ankle and foot posture, which in turn, will affect the foot and ankle muscle activity. Therefore, we measured muscle activity of the ankle muscles (TA and PL) as well as the foot intrinsic muscle (AbdH) during SFE with or without IHA. In particular, pes planus subjects have been reported to overuse the TA due to a weakened AbdH to maintain the MLA. ${ }^{10}$ Furthermore, previous researchers measured $\mathrm{AbdH}$ to compare foot intrinsic muscle activity, 2,10,12,16 which has been described as a muscle that plays an important role in supporting the MLA and controlling foot pronation in a static stance. ${ }^{2,17}$ The purpose of this study was to compare the effects of IHA and exercise posture on the muscle activity of the AbdH, TA, and PL, as well as the change in the MLA angle during SFE in participants with pes planus. We hypothesized that, during SFE with IHA, the AbdH muscle activity and the change in the MLA angle will be greater than SFE without IHA. We also hypothesized that, during SFE, with or without IHA, there will be a difference in the $\mathrm{AbdH}$ and ankle muscle activity, as well as in the change in the MLA angle, according to the exercise position (sitting or standing).

\section{Method}

\section{Study Design}

This study was based on 2-way repeated analyses of variance with 2 within-participant factors (IHA condition and exercise posture). The AbdH, TA, PL, and gluteus medius (Gmed) surface electromyography (EMG) activity, as well as the MLA angle during 4 different SFE, were measured in participants with pes planus.

\section{Participants}

A power analysis was performed using the G*Power software (version 3.1.2; Franz Faul, University of Kiel, Kiel, Germany). The necessary sample size of 15 participants to achieve a power of 0.80 , an effect size of 0.51 , and an alpha level of .05 was calculated from data obtained from a pilot study of 6 participants. The participants were selected based on the presence of pes planus as determined by the navicular drop test. The navicular drop was measured twice, and only participants with mean measurements greater than $10 \mathrm{~mm}$ were invited to participate in this study. ${ }^{18}$ The study included 32 pes planus participants (21 males and 11 females) with an age of 22.9 (1.98) (range, 20-28) years, height of $169.18(7.02) \mathrm{cm}$, weight of $68.0(11.9) \mathrm{kg}$, body mass index of $23.55(3.17) \mathrm{kg} / \mathrm{m}^{2}$, and a navicular drop of 11.31 (1.41) mm. Participants with past or current inflammatory arthritis; foot ankle surgery; diabetes; or toe deformities, such as claw toe, hallux valgus, or hammertoe, were excluded. ${ }^{10}$ Participants who failed to correctly perform SFE during the familiarization sessions or those who had pain or discomfort during the measurements were also excluded. The procedures were explained in detail to the participants prior to the study, and all participants signed an informed consent form before participating in the study. This study was approved by the Yonsei University Mirae Institutional Review Board.

\section{Procedures}

Before the measurements were taken, the principal researcher demonstrated the SFE, with or without IHA, for approximately 30 minutes in order to familiarize the participants with the program. During the familiarization session, the increase in $\mathrm{AbdH}$ muscle activity was monitored in order to confirm the correct performance of the SFE. All participants were blinded to both the results and the intention of the experiment. The 4 types of SFE were randomly performed using Microsoft Excel (Microsoft Corp, Redmond, WA). The participants performed 3 trials of each exercise, with a 5-second hold to measure the muscle activity of the AbdH, TA, PL, and Gmed, as well as the MLA angles. ${ }^{10}$ In order to minimize the test effects and fatigue, a 10-minute rest was allowed between each exercise. ${ }^{2}$ The collected EMG amplitudes of the AbdH, TA, PL, and Gmed muscles during all types of SFE were recorded as percentages of mean maximal voluntary isometric contraction (\%MVIC).

SFE Without IHA in a Sitting Position (SF-SIT). The participants were asked to place their feet in a wooden box and sit on a heightadjustable table; the height of the table was adjusted so that the participant's hip and knee were approximately $90^{\circ}$. The width between both feet was determined to be equal to the pelvic width by the researcher. Each second toe and patellar bone was aligned in order to maintain a constant leg position during the SFE. During the measurements, the participant was asked to sit correctly, keeping the spine upright and the pelvis neutral. The participants were asked to pull the first metatarsal head toward their heel in order to shorten their foot. During the SFE, the participants' toes were not bent, and their forefoot and heel were kept in contact with the wooden box (Figure 1A).

SFE Without IHA in a Standing Position (SF-STD). The participant was placed by the researcher in the standing position so that the width between both feet was equal to the width of the pelvis and the second toe was aligned with the patella. During the SFE, the participant was asked to position the spine straight, maintain the pelvis in a neutral position, and place the centerline of the body midway between the feet. The subjects were given feedback from the researchers to assist in the maintenance of accurate body alignment (Figure 1B).

SFE With IHA in a Sitting Position (SFIHA-SIT). The starting position of the SFE with IHA in the sitting position was the same as that of the SFE alone in the sitting position, except that an elastic band was placed above the knee to provide resistance of the hip abductor muscles. In order to provide relative resistance for each participant, the elastic band was adjusted to a length that allowed the hip abduction in a sitting position to be performed 10 times or less. ${ }^{19}$ To maintain a constant hip joint angle during the exercise, the second toe and patellar bone were kept aligned using a target bar. The participants performed SFE while abducting the hip joint against the elastic band and keeping the fibular head in contact with the target bar (Figure 1C).

SF With IHA in a Standing Position (SFIHA-STD). The starting position was set by the researcher under the same conditions as the 


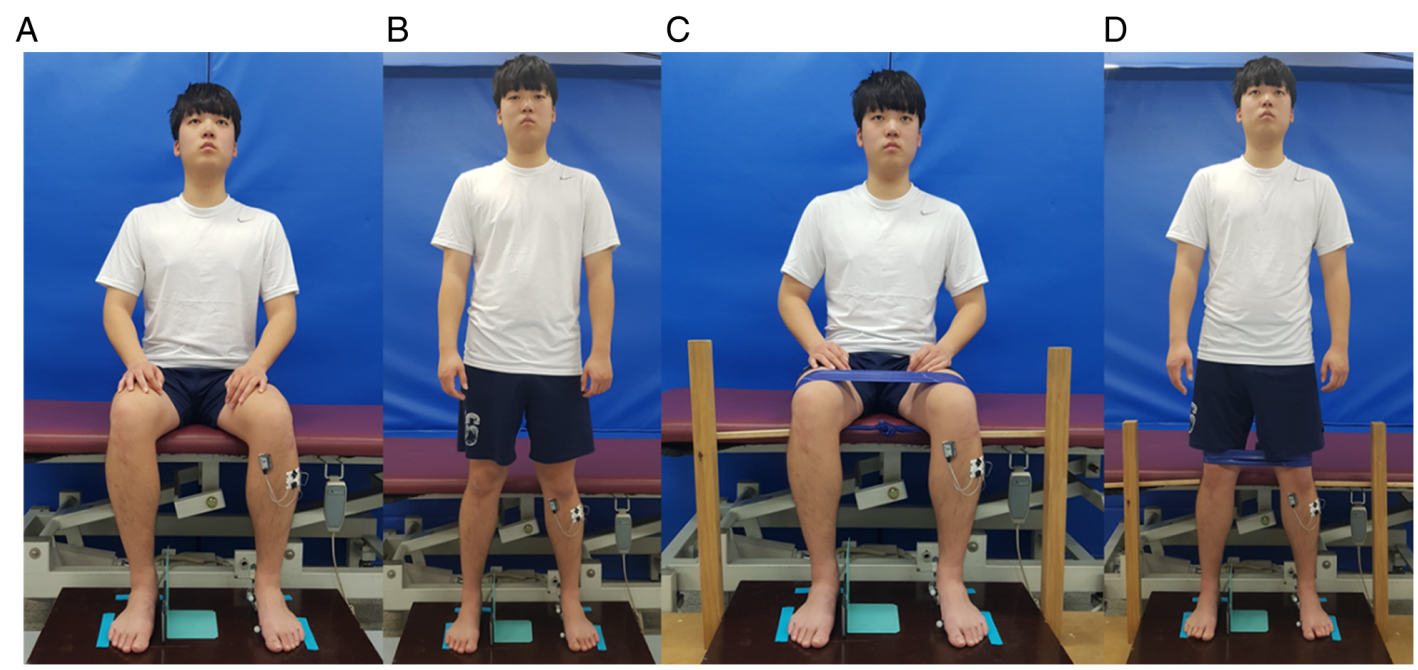

Figure 1 - SFE with or without IHA in a sitting and standing position. (A) SF-SIT, (B) SF-STD, (C) SFIHA-SIT, (D) SFIHA-STD. IHA indicates isometric hip abduction; SF-SIT, SFE without IHA in a sitting position; SF-STD, SFE without IHA in a standing position; SFE, short-foot exercise; SFIHA-SIT, SFE with IHA in a sitting position; SFIHA-STD, SFE with IHA in a standing position.

SFE, only in a standing position, except that an elastic band was tied above the knee joint to provide resistance to the hip abduction. The length of the elastic band was adjusted so that the hip abduction was able to be performed fewer than 5 times in a sitting position and to provide sufficient elastic resistance to the participants during SFE in the standing position. The participants were kept in contact with the fibular head of the target bar to maintain a constant position during SFE (Figure 1D).

\section{Surface EMG Data Collection}

Surface EMG (Noraxon TeleMyo DTS Wireless System; Noraxon Inc, Scottsdale, AZ) was used to measure the activity of the AbdH, PL, TA, and Gmed. The sampling rate of the EMG signal was set at $1000 \mathrm{~Hz}$. The raw signal was filtered using a digital band-pass filter at 20 to $450 \mathrm{~Hz}$ and the notch filter at $60 \mathrm{~Hz}$. The measured data were analyzed using Noraxon MyoResearch XP software (version 1.08; Noraxon Inc, Scottsdale, AZ). The electrode sites were prepared by shaving and cleansing the skin with cotton dipped in alcohol in order to minimize output impedance for the EMG signal and to allow for proper electrode attachment. Disposable $\mathrm{Ag} / \mathrm{AgCl}$ surface electrodes were placed approximately $2 \mathrm{~cm}$ apart in the direction of the muscle fibers. A detailed description of the electrode attachment area is as follows. ${ }^{20}$ the EMG electrodes for the $\mathrm{AbdH}$ were attached approximately 1 to $2 \mathrm{~cm}$ posterior to the navicular tuberosity, located just in front of the vertical line from the anterior margin of the medial malleolus. ${ }^{17}$ The electrodes of the TA were attached at the one-third point between the tip of the fibular head and the medial malleolus. The PL electrodes were placed at $25 \%$ on the line between the tip of the head of the fibula to the tip of the lateral malleolus. The electrodes for the Gmed measurements were attached at the midpoint between the iliac crest and the greater trochanter. The MVIC was measured to normalize all EMG signals. The MVIC of each muscle was measured using standard manual muscle testing positions, as recommended by Kendall et al. ${ }^{21}$ To measure the MVIC of the AbdH muscle, the researcher fixed the heel firmly at a neutral ankle position and put resistance on the medial side of the first metatarsal and proximal phalanx in order to produce abduction of the metatarsophalangeal joint of the great toe. The participant was asked to adduct the hallux and forefoot against the applied resistance. To measure the MVIC of TA, the participant performed maximal ankle dorsiflexion against the resistance applied to the dorsomedial aspect of the foot. The MVIC of the PL was tested with the ankle in the neutral position, and the participants performed ankle eversion and plantar flexion against the resistance applied to the lateral and plantar surface of the foot. The MVIC of the Gmed was measured in a side-lying position on the treatment table, with the test leg up, the bottom hip flexed at $45^{\circ}$, and the knee flexed at $90^{\circ}$ for stability. The participant performed maximal hip abduction against the resistance applied to the ankle. The EMG data were measured for 5 seconds, and the middle 3 seconds were used to determine the mean MVIC amplitude. In order to minimize the effect of fatigue, the participants had a 1-minute break between MVIC measurements. The collected EMG activities of the AbdH, TA, PL, and Gmed muscles during the SFE were described as \%MVIC.

\section{MLA Calculation}

For the MLA angle measurement, the researcher kept the subtalar joint neutral and then attached reflective markers on 3 anatomical points (the navicular tuberosity, the medial aspect of the first metatarsal head, and the medial side of the calcaneal bone) of the foot. The attachment point of the calcaneal marker was $30 \mathrm{~mm}$ (women) or $40 \mathrm{~mm}$ (men) from the most posterior part of the calcaneus and $30 \mathrm{~mm}$ (women) or $35 \mathrm{~mm}$ (men) from the surface of the wooden box..$^{22}$ The camera was placed $20 \mathrm{~cm}$ from the medial side of the measurement foot in order to take images of the reflective markers during the SFE (Figure 2). In order to minimize disturbance to the SFE, we used a Bluetooth remote controller while shooting with a smartphone camera. Image J software (US National Institutes of Health, Bethesda, MD) was used to measure the MLA angle through digital images taken during the SFE and resting state. ${ }^{10}$ The researcher set the point based on the 3 reflective markers of the digital image, and the MLA angle was calculated using Image J. To examine intrarater reliability, a rater with over 10 years' experience as a clinical therapist measured the 
MLA angle twice, using ImageJ software for all participants. The difference in the MLA angle between the resting state and the SFE was obtained, and the MLA angle change value was used for statistical analysis. ${ }^{12}$

\section{Statistical Analysis}

The statistical package for the social sciences software (version 20; SPSS Inc, Chicago, IL) was used for statistical analysis. Two-way repeated analyses of variance with 2 within-participant factors (exercise type: with and without IHA; exercise position: sitting and standing) were used to determine the statistical significance of the AbdH, TA, PL, and Gmed activity, as well as the change in MLA angle. The level of significance was set at .05. If a no significant exercise type $\times$ exercise position interaction was revealed from the 2-way repeated analyses of variance, the main effects of the exercise type and exercise position were determined. If a significant exercise type $\times$ exercise position interaction was found, a pair-wise comparison with Bonferroni correction was used to determine the simple effect. To determine the intrarater reliability of the MLA angle measurement using Image $J$, the intraclass correlation coefficient $\left(\mathrm{ICC}_{3,1}\right)$ and the standard error of measurements (SEM) were used based on all participants, for each exercise in both the sitting and standing positions.

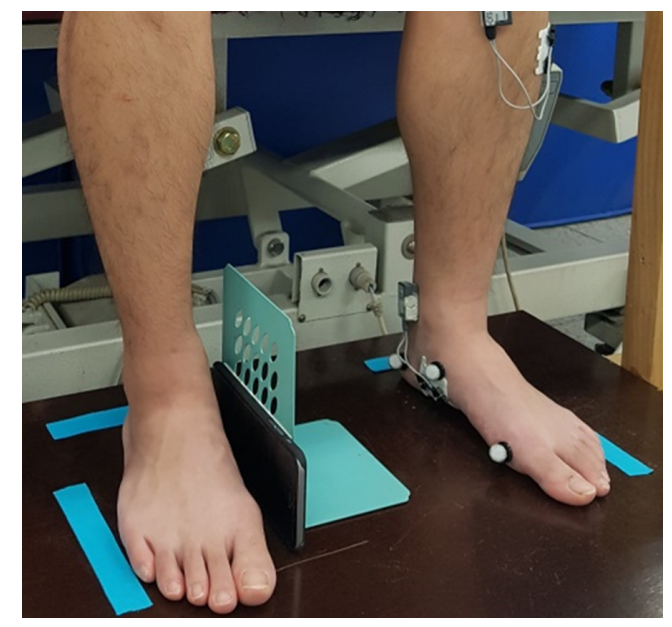

Figure 2 - Measurement of the medial longitudinal arch angle.

A

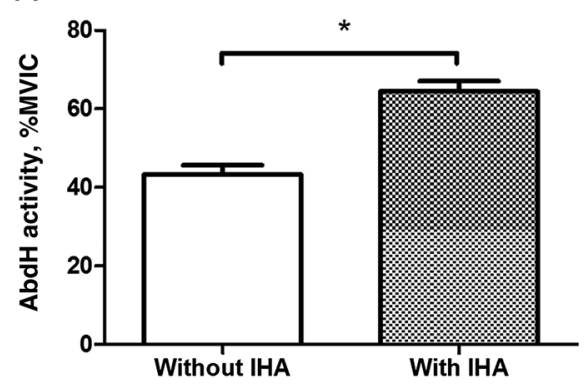

\section{Results}

\section{AbdH, TA, PL, and Gmed Muscle Activity}

There was no significant exercise type $\times$ exercise position interaction in terms of the muscle activity of the $\mathrm{AbdH}\left(F_{1,31}=0.73, P=\right.$ $.79)$; TA $\left(F_{1,31}=1.45, P=.24\right)$; and $\operatorname{Gmed}\left(F_{1,31}=0.98, P=.33\right)$. A significant exercise type $\times$ exercise position interaction was observed in terms of PL activity $\left(F_{1,31}=6.86, P<.01\right)$.

The AbdH activity was a significant main effect of the exercise type $\left(F_{1,31}=120.07, P<.01\right)$ and exercise position $\left(F_{1,31}=20.45\right.$, $P<.01)$. The EMG activity of $\mathrm{AbdH}$ was significantly greater with IHA than without IHA (64.46\%MVIC with IHA and 43.20 $\%$ MVIC without IHA; Figure 3A). The EMG activity of AbdH was also significantly increased in the standing position compared with the sitting position $(60.28 \%$ MVIC in the standing position and $47.37 \%$ MVIC in the sitting position; Figure 3B).

The TA activity was a significant main effect of the exercise type $\left(F_{1,31}=15.64, P<.01\right)$, but there was no significant main effect of exercise position $\left(F_{1,31}=3.95, P=.07\right)$. The TA activity was significantly lower with IHA than without IHA $(6.45 \%$ MVIC with IHA and 15.29\%MVIC without IHA) (Figure 4A).

The PL activity was significantly greater in the SFIHA-STD than in the SFIHA-SIT $(P<.01 ; 95 \%$ confidence interval $[C I]$, 11.58-25.61; effect size $[\mathrm{ES}]=1.1)$, and the EMG activity of the PL was significantly greater during the SF-STD than during the SFE without IHA in a sitting position $(P<.01 ; 95 \% \mathrm{CI}, 2.85-$ 16.07; ES = 0.46) (Figure 5).

The Gmed activity was a significant main effect of the exercise position $\left(F_{1,31}=11.34, P<.01\right)$, but there was no significant main effect of the exercise type $\left(F_{1,31}=0.002, P=.97\right)$. The Gmed activity was significantly greater in the standing position than the sitting position (12.35\%MVIC in standing and $7.66 \% \mathrm{MVIC}$ in sitting; Figure 4B).

\section{Change in MLA Angle}

The measurements of the MLA angle showed high intrarater reliability in the SFE without IHA ( $\mathrm{ICC}_{3,1} ; 95 \% \mathrm{CI}, .92$ [.85-.96]; $\mathrm{SEM}=2.37$ in the sitting position; $\mathrm{ICC}_{3,1} ; 95 \% \mathrm{CI}$, .96 [.93-.85]; $\mathrm{SEM}=1.40$ in the standing position) and the SFE with IHA $\left(\mathrm{ICC}_{3,1} ; 95 \% \mathrm{CI}, .91\right.$ [.82-.95]; SEM $=2.53$ in the sitting position; $\mathrm{ICC}_{3,1} ; 95 \% \mathrm{CI}, .96$ [.94-.98]; SEM $=1.77$ in the standing position). The change in MLA angle had a significant exercise type $\times$ exercise posture interaction $\left(F_{1,31}=15.58, P<.01\right)$. The change in

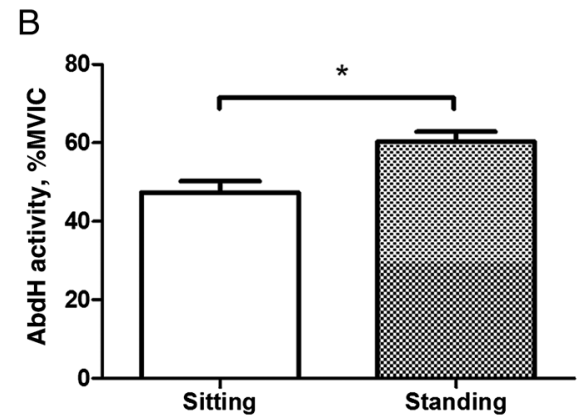

Figure 3 - (A) Comparison of AbdH muscle activity during the SFE with and without IHA, (B) Comparison of AbdH muscle activity during the SFE in a sitting and standing position. \%MVIC indicates percentage of maximal voluntary isometric contractions; AbdH, abductor hallucis; IHA, isometric hip abduction; SFE, short-foot exercise. ${ }^{*} P<.05$, significant difference in main effect. 
A

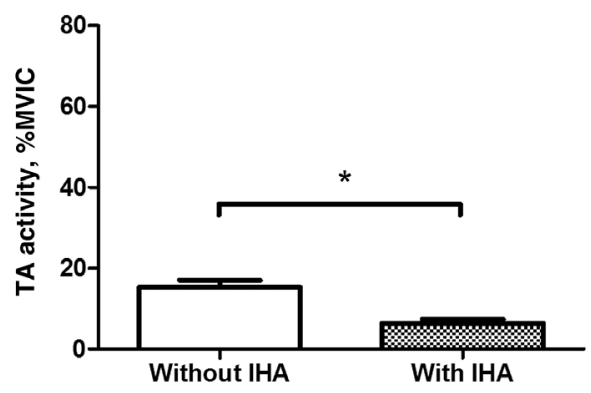

B

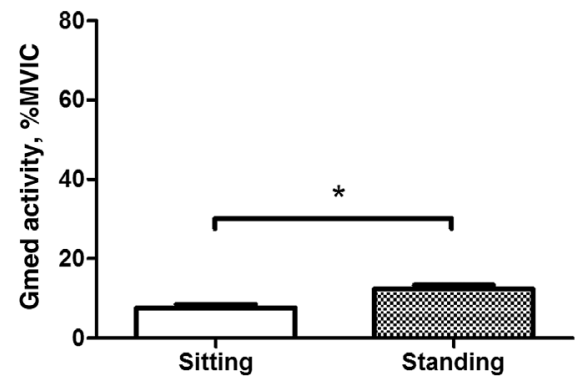

Figure 4 - (A) Comparison of TA muscle activity during the SFE with and without IHA, (B) Comparison of Gmed muscle activity during the SFE in a sitting and standing position. \%MVIC indicates percentage of maximal voluntary isometric contractions; Gmed, gluteus medius; IHA, isometric hip abduction; SFE, short-foot exercise; TA, tibialis anterior. ${ }^{*} P<.05$, significant difference in main effect.

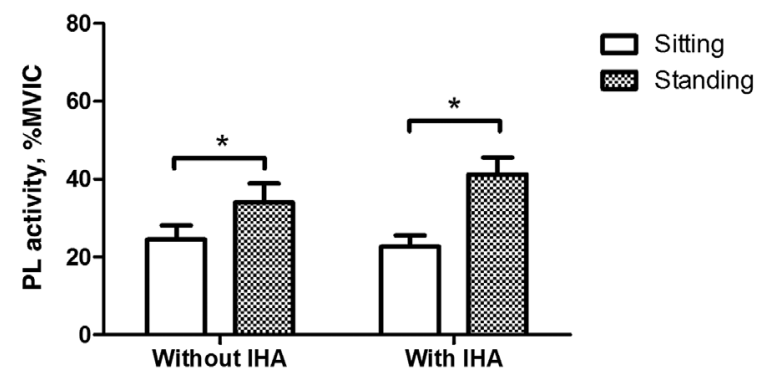

Figure 5 - Comparisons of PL activity during the 4 SFE (SFE without IHA in a sitting position, SFE without IHA in a standing position, SFE with IHA in a sitting position, and SFE with IHA in a standing position). $\%$ MVIC indicates percentage of maximal voluntary isometric contractions; IHA, isometric hip abduction; PL, peroneus longus; SFE, short-foot exercise. $* P<.01$, significant difference in simple effect.

the MLA angle was significantly greater in the SFIHA-STD than the SF-STD $(P<.01 ; 95 \%$ CI, 3.48-5.16; ES $=1.23)$ and SFIHASIT $(P<.01 ; 95 \% \mathrm{CI}, .76-3.69 ; \mathrm{ES}=0.55)$. The change in the MLA angle in the SFIHA-SIT was greater than that in the SFE without IHA in a sitting position $(P<.01 ; 95 \% \mathrm{CI}, 1.18-2.80$; $\mathrm{ES}=0.49)($ Figure 6).

\section{Discussion}

We investigated whether the AbdH and ankle muscle activity, as well as the MLA angle, could be changed with the incorporation of IHA during SFE performed by participants with pes planus in sitting and standing positions. Significant interactions between the exercise type and exercise position were only observed in the PL muscle activity and in the change in the MLA angle. Moreover, the muscle activity of the AbdH was significantly increased with IHA compared to without it, and it was significantly increased in the standing position compared with sitting. The TA muscle activity in both the sitting and standing positions was significantly lower with IHA than without, and the Gmed muscle activity was significantly higher in the standing position compared with sitting. To the best of our knowledge, this is the first study to investigate a strategy to increase AbdH activity and decrease activation of the TA in participants with pes planus during SFE.

Our results demonstrated significantly higher AbdH activity during SFE with IHA than without it (mean difference $=32.98$

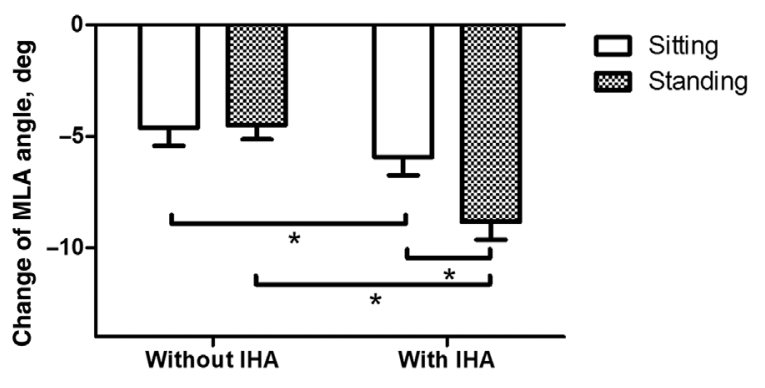

Figure 6 - Comparisons of the change in MLA angle during the 4 SFE (SFE without IHA in a sitting position, SFE without IHA in a standing position, SFE with IHA in a sitting position, and SFE with IHA in a standing position). \%MVIC indicates percentage of maximal voluntary isometric contractions; IHA, isometric hip abduction; MLA, medial longitudinal arch; SFE, short-foot exercise. ${ }^{*} P<.01$, significant difference in simple effect.

\%MVIC); this result supports our research hypothesis and has several interpretations. First, the IHA may have contributed to the increase in AbdH muscle activity by restricting the compensatory movement of the hip joint during the SFE. A previous investigation reported that excessive pronation of the foot during the stance phase may result in an increased internal rotation of the tibia and hip, thereby increasing hip adduction. ${ }^{23}$ Similarly, during the SFE, participants with pes planus may be able to perform adduction or internal rotation of the hip joint in order to compensate for the weakened AbdH. The IHA not only enhances the stability of the pelvis and trunk, ${ }^{24,25}$ but also contributes to the improvement of hip joint stability by sustaining IHA during SFE. Therefore, accurately performed SFE without compensatory action due to IHA would have affected the increase in AbdH muscle activity. Second, the IHA would have affected the alignment of the foot during SFE. The alignment of the foot in an excessive pronated state causes the intrinsic muscle to become weakened and stretched, and this state of malalignment may lead to difficulty in generating an appropriate or optimal force.${ }^{16}$ During the SFE, the IHA may cause an external rotation of the tibia and calcaneal supination, and the forefoot and midfoot are simultaneously twisted into relative pronation in order for the metatarsophalangeal joint to remain in contact with the ground. Subsequently, the subtalar joint may supinate, and the MLA may become tense and elevated. ${ }^{26}$ Therefore, the participants performed SFE in the well-aligned foot with an elevated MLA. The results of our study support this interpretation. In our study, the 
change in the MLA angle in both positions was significantly greater with IHA than without it. In addition, our study showed that the AbdH muscle activity was greater than $60 \%$ of the MVIC during SFE with IHA. According to a previous study, an MVIC activation level greater than $60 \%$ is sufficient for muscle strengthening. ${ }^{27}$ Therefore, SFE with IHA can also be applied as an AbdH musclestrengthening program.

We also found that the EMG activity of the AbdH was significantly increased in the standing position compared with the sitting position (mean difference $=21.45 \%$ MVIC). Indeed, previous researchers have demonstrated that the AbdH plays an important role in supporting the MLA and controlling pronation in a static stance. ${ }^{17}$ In addition, several investigators have reported that when the weight load on the foot increases, such as in standing or walking, the intrinsic foot muscle activity also increases. ${ }^{2}$ Therefore, the AbdH muscle activity was significantly increased in the standing position with greater weight on the foot than in the sitting position.

In our study, the TA muscle activity was significantly lower with IHA than without it (mean difference $=57.56 \%$ MVIC), and there was no significant difference in terms of the exercise position. During the SFE, the use of IHA reduced the TA muscle activity, while the AbdH muscle activity increased. This finding is in agreement with the results of Lee et al, ${ }^{10}$ who compared the $\mathrm{AbdH}$ and TA muscle activity between pes planus participants and neutral foot participants during SFE and reported a significantly lower activity of $\mathrm{AbdH}$ and a significantly higher TA/AbdH ratio in pes planus participants than neutral foot participants due to the compensatory role of the TA muscle. Lee et $\mathrm{al}^{10}$ suggested that a new method should be developed whereby AbdH muscle activity is increased as TA activity is decreased because of the possibility of additional musculoskeletal disorders if compensatory overuse of the TA continues in pes planus participants. Other researchers have reported that the SFE in the ankle plantarflexion position can significantly decrease the TA activity compared with SFE in the neutral ankle position; however, they reported no significant difference in AbdH muscle activity. ${ }^{12}$ When the SFE was performed in the alignment of the foot with the MLA already elevated by the IHA, the TA would not have to compensate to increase the MLA. Thus, the IHA may increase the AbdH muscle activity by reducing the compensatory muscle contraction of the TA.

According to our study, the PL muscle activity was significantly different in the exercise position, but there was no significant difference between the presence or absence of IHA in the same position. The PL muscles are most activated in the weight-bearing state, such as during a heel raise and the mid-to-late stance phase of walking, and the PL assist other plantar flexor muscles with plantar flexion at the talocrural joint. ${ }^{26}$ Therefore, since the standing position leads to a greater load on the feet than the sitting posture, the muscle activity of the PL will also be greater.

In this study, the Gmed muscle activity was significantly greater in the standing position than the sitting position (mean difference $=37.97 \%$ MVIC). The Gmed prevents the opposite side of the pelvis from dropping during the stance phase of gait, and it has a major role in providing frontal stability for the pelvis during walking and other functional activities. ${ }^{26}$ Therefore, the Gmed muscle activity increased significantly in the standing position, with a greater load on the hip joint than in the sitting position.

The results of this study supported our hypothesis that, during SFE in both positions, the use of IHA would increase the change in the MLA angle compared with SFE alone. The change in the MLA angle was significantly higher in the SFIHA-STD compared with the SFIHA-SIT $\left(\right.$ mean difference $=2.23^{\circ}$ ) and SF-STD (mean difference $=4.32^{\circ}$ ), which seems to be related to the results of the 2 main effects of AbdH activity. Furthermore, the AbdH muscle activity was significantly greater in the standing position than the sitting position, and with IHA, it was significantly greater than without IHA. The change in the MLA angle was also significantly higher in the SFIHA-STD when the standing position and IHA conditions were simultaneously applied. The results of our study are consistent with a previous study, and this study also reported that the $\mathrm{AbdH}$ muscle activity contributed to a decrease in the MLA angles. ${ }^{2}$

Our study had several limitations. First, we did not measure the activity of other hip abductor muscles due to difficulties in measuring these muscles using surface EMG. Other hip abductor muscles, such as the deep lateral rotators, may activate during SFE with IHA. Second, we could not measure 3-dimensional motion, such as a transverse arch or calcaneal pronation, which may occur during SFE, because the MLA angle was only measured in 2 dimensions. Third, since this study was cross-sectional, the long-term effects of the 4 different conditions of SFE on the activity of AbdH and ankle muscles, as well as the MLA angle, could not be determined. Thus, further studies should be conducted in order to evaluate the long-term effects of SFE with IHA on the strength of intrinsic and extrinsic foot-muscles, the change of MLA angle, and functional activities.

\section{Conclusions}

We compared the effects of IHA on the activity of AbdH and ankle muscles, as well as the change in the MLA angle, during SFE. The change in the MLA angle was significantly higher in the SFIHASTD than in the other SFE conditions. Furthermore, the performance of IHA during SFE significantly increased the AbdH muscle activity, while the TA muscle activity was significantly lower. The observation of more than a 60\% MVIC in AbdH muscles during SFE with IHA may be applicable, not only as an increase in AbdH activation, but also as a muscle-strengthening program. Therefore, SFE with IHA may represent a new strengthening program for weakened intrinsic foot muscles after sports injury, as well as in rehabilitation programs for patients with pes planus who overuse TA muscles and require enhanced activation of intrinsic foot muscles.

\section{Acknowledgments}

The authors would gratefully like to thank all voluntary participants who took part in this study. No external funds were supplied for this study. The equipment used in this study is not being endorsed by the authors.

\section{References}

1. Sahrmann S. Movement System Impairment Syndromes of the Extremities, Cervical and Thoracic Spines. St. Louis, MO: Elsevier/Mosby; 2011.

2. Jung DY, Kim MH, Koh EK, Kwon OY, Cynn HS, Lee WH. A comparison in the muscle activity of the abductor hallucis and the medial longitudinal arch angle during toe curl and short foot exercises. Phys Ther Sport. 2011;12(1):30-35. PubMed ID: 21256447 doi:10.1016/j.ptsp.2010.08.001 
3. Mann R, Inman VT. Phasic activity of intrinsic muscles of the foot. $J$ Bone Joint Surg Am. 1964;46(3):469-481. PubMed ID: 14131426 doi:10.2106/00004623-196446030-00001

4. Martin RL, Davenport TE, Reischl SF, et al. Heel pain-plantar fasciitis: revision 2014. J Orthop Sports Phys Ther. 2014;44(11): A1-A33. PubMed ID: 25361863 doi:10.2519/jospt.2014.0303

5. Easley ME, Trnka HJ. Current concepts review: hallux valgus part 1: pathomechanics, clinical assessment, and nonoperative management. Foot Ankle Int. 2007;28(5):654-659. PubMed ID: 17559782 doi:10. 3113/FAI.2007.0654

6. Ryan M, Grau S, Krauss I, Maiwald C, Taunton J, Horstmann T. Kinematic analysis of runners with Achilles mid-portion tendinopathy. Foot Ankle Int. 2009;30(12):1190-1195. PubMed ID: 20003878 doi:10.3113/FAI.2009.1190

7. Powers CM, Maffucci R, Hampton S. Rearfoot posture in subjects with patellofemoral pain. J Orthop Sports Phys Ther. 1995;22(4): 155-160. PubMed ID: 8535473 doi:10.2519/jospt.1995.22.4.155

8. William PE. Rehabilitation Techniques for Sports Medicine and Athletic Training. New York, NY: McGraw-Hill; 2004.

9. Anderson MK, Hall SJ, Martin M. Foundations of Athletic Training: Prevention, Assessment, and Management. Philadelphia, PA: Lippincott Williams \& Wilkins; 2004.

10. Lee JH, Cynn HS, Yoon TL, Choi SA, Kang TW. Differences in the angle of the medial longitudinal arch and muscle activity of the abductor hallucis and tibialis anterior during sitting short-foot exercises between subjects with pes planus and subjects with neutral foot. J Back Musculoskelet Rehabil. 2016;29(4):809-815. PubMed ID: 27002666 doi:10.3233/BMR-160693

11. Moon DC, Kim K, Lee SK. Immediate effect of short-foot exercise on dynamic balance of subjects with excessively pronated feet. $J$ Phys Ther Sci. 2014;26(1):117-119. PubMed ID: 24567688 doi:10.1589/ jpts.26.117

12. Yoon H-B, Kim J-H, Park J-H, Jeon H-S. Comparison of the foot muscle EMG and medial longitudinal arch angle during short foot exercises at different ankle position. Phys Ther Korea. 2017;24(4): 46-53. doi:10.12674/ptk.2017.24.4.046

13. Powers CM. The influence of abnormal hip mechanics on knee injury: a biomechanical perspective. J Orthop Sports Phys Ther. 2010; 40(2):42-51. PubMed ID: 20118526 doi:10.2519/jospt.2010.3337

14. Snyder KR, Earl JE, O’Connor KM, Ebersole KT. Resistance training is accompanied by increases in hip strength and changes in lower extremity biomechanics during running. Clin Biomech. 2009; 24(1):26-34. doi:10.1016/j.clinbiomech.2008.09.009

15. Beckman SM, Buchanan TS. Ankle inversion injury and hypermobility: effect on hip and ankle muscle electromyography onset latency. Arch Phys Med Rehabil. 1995;76(12):1138-1143. PubMed ID: 8540791 doi:10.1016/S0003-9993(95)80123-5
16. Lee J, Yoon J, Cynn H. Foot exercise and taping in patients with patellofemoral pain and pronated foot. J Bodyw Mov Ther. 2017; 21(1):216-222. PubMed ID: 28167183 doi:10.1016/j.jbmt.2016. 07.010

17. Fiolkowski P, Brunt D, Bishop M, Woo R, Horodyski M. Intrinsic pedal musculature support of the medial longitudinal arch: an electromyography study. J Foot Ankle Surg. 2003;42(6):327-333. PubMed ID: 14688773 doi:10.1053/j.jfas.2003.10.003

18. Cote KP, Brunet ME, Gansneder BM, Shultz SJ. Effects of pronated and supinated foot postures on static and dynamic postural stability. $J$ Athl Train. 2005;40(1):41-46. PubMed ID: 15902323

19. Decker MJ, Hintermeister RA, Faber KJ, Hawkins RJ. Serratus anterior muscle activity during selected rehabilitation exercises. Am J Sports Med. 1999;27(6):784-791. PubMed ID: 10569366 doi:10.1177/03635465990270061601

20. SENIAM. Project-sensor placement. 2018. Available at http://www. seniam.org. Accessed December 11, 2018.

21. Kendall FP, McCreary EK, Provance PG, Rodgers M, Romani WA. Muscles: Testing and Function, With Posture and Pain: Includes a Bonus Primal Anatomy. Vol. 5. Philadelphia, PA: Lippincott Williams \& Wilkins; 2005:158.

22. Bandholm T, Boysen L, Haugaard S, Zebis MK, Bencke J. Foot medial longitudinal-arch deformation during quiet standing and gait in subjects with medial tibial stress syndrome. J Foot Ankle Surg. 2008;47(2):8995. PubMed ID: 18312915 doi:10.1053/j.jfas.2007.10.015

23. Tiberio D. The effect of excessive subtalar joint pronation on patellofemoral mechanics: a theoretical model. J Orthop Sports Phys Ther. 1987;9(4):160-165. PubMed ID: 18797010 doi:10. 2519/jospt.1987.9.4.160

24. Kang MH, Kim SY, Kang MJ, Yoon SH, Oh JS. Effects of isometric hip movements on electromyographic activities of the trunk muscles during plank exercises. J Phys Ther Sci. 2016;28(8):2373-2375. PubMed ID: 27630435 doi:10.1589/jpts.28.2373

25. Stevens VK, Vleeming A, Bouche KG, Mahieu NN, Vanderstraeten GG, Danneels LA. Electromyographic activity of trunk and hip muscles during stabilization exercises in four-point kneeling in healthy volunteers. Eur Spine J. 2007;16(5):711-718. PubMed ID: 16896840 doi:10.1007/s00586-006-0181-1

26. Neumann DA. Kinesiology of the Musculoskeletal System: Foundations for Rehabilitation. St. Louis, MO: Elsevier Health Sciences; 2013.

27. Digiovine NM, Jobe FW, Pink M, Perry J. An electromyographic analysis of the upper extremity in pitching. J Shoulder Elbow Surg. 1992;1(1):15-25. PubMed ID: 22958966 doi:10.1016/S10582746(09)80011-6 